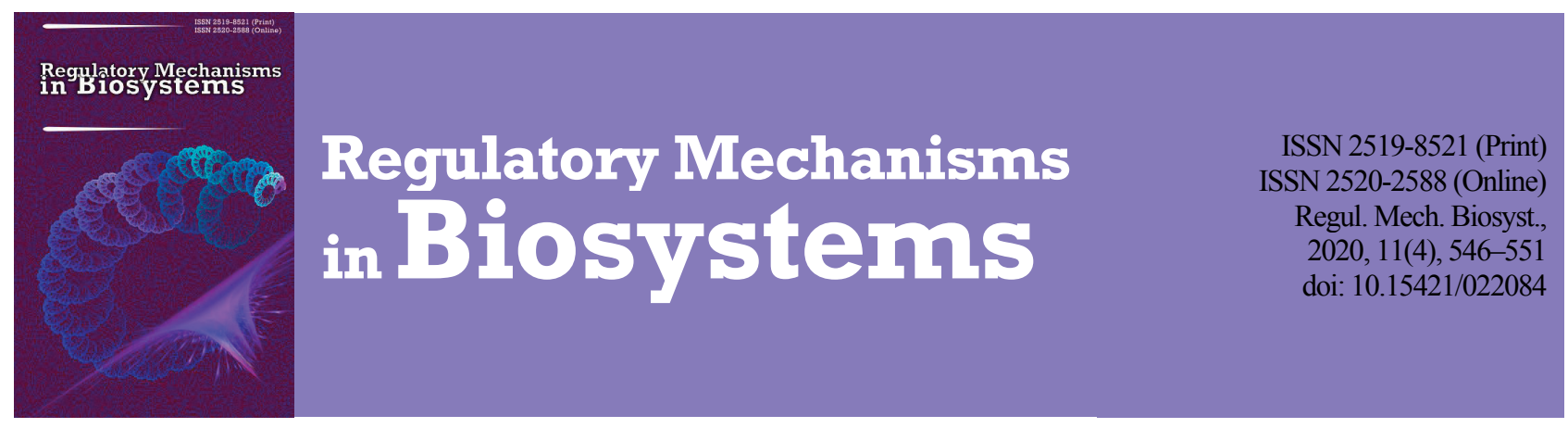

\title{
Features of neurosteroid support of the state of alcohol dependence and its correction with dosed physical load in rats
}

\author{
A. M. Titkova, O. G. Berchenko, O. V. Veselovska, A. V. Shliakhova \\ Institute of Neurology, Psychiatry and Narcology of NAMS of Ukraine, Kharkiv, Ukraine
}

Article info

Received 10.11.2020

Received in revised form

05.12.2020

Accepted 07.12.2020

Institute of Neurology, Psychiatry and Narcolog of NAMS of Ukraine,

Academic Pavlov st., 46

Kharkiv, 61068, Ukraine.

Tel.: + 380963804258 .

E-mail:

annatitkova2@ukr.net

\begin{abstract}
Titkova, A. M., Berchenko, O. G., Veselovska, O. V., \& Shliakhova, A. V. (2020). Features of neurosteroid support of the state of alcohol dependence and its correction with dosed physical load in rats. Regulatory Mechanisms in Biosystems, 11(4), 546-551. doi:10.15421/022084
\end{abstract}

The role of steroid hormones in regulation of the functions of the emotiogenic limbic-neocortical system has been actively studied over the recent decades in order to determine their synthesis in the brain structures and role in the development and maintenance of dependence on psychoactive substances. However, the wide range of neurosteroids and their metabolites, as well as structural specific features of the synthesis of both neurohormones and their receptors make it difficult to obtain experimental data and interpret the results of the study. The participation of progesterone, cortisol, testosterone and estradiol in the development of alcohol dependence and the changes in their concentrations in the hypothalamus, hippocampus, amygdala and serum under the influence of dosed physical load were studied in 48 outbred adult male rats. Alcohol dependence was modeled by means of consuming food containing alcohol in the dose of $1.25 \mathrm{~g}$ of ethanol per $1 \mathrm{~kg}$ of rat body weight for two months. Dosed physical load was reproduced by a rat running in a wheel for 30 minutes daily for 7-10 days against the background of alcohol withdrawal. Neuroethological testing of craving for alcohol, EEG recording of the neocortex, hippocampus and amygdala was performed using a computer-diagnostic complex. The concentration of steroid hormones was determined in the structures of the brain and blood serum by the enzyme-linked immunosorbent assay. It was shown that dosed physical load attenuated the alcohol motivation of rats. On the 5th day it suppressed the electrographic manifestations of paroxysmal activity in the hippocampus and increased the level of the theta-rhythm in the amygdala, and on the 7th day it activated the neocortex with increasing beta-rhythm. This effect was accompanied by an increase in serum testosterone level against the background of maintaining functional tension of the peripheral glucocorticoid link of the hypothalamus-pituitary-adrenal system, which was observed in a state of alcohol dependence. The study demonstrated that progesterone plays the key role in allostatic rearrangements of the functional state of animals. An imbalance of progesterone levels was revealed in the brain structures: an increase - in the hypothalamus and hippocampus, and a decrease - in the amygdala under alcohol dependence; a decrease - in the hippocampus with recovery in the amygdala against the background of its high level in the hypothalamus, which occurs under the influence of dosed physical load on the rats under alcohol withdrawal. Thus, the dosed physical load is a promising approach to alcohol dependence rehabilitation.

Keywords: steroid hormones; electrical activity; brain; alcohol withdrawal; running wheel; rats.

\section{Introduction}

The effects of substances with addictogenic potential are realized on the basis of the intracerebral system of positive reinforcement in which the leading role belongs to the mesolimbic dopaminergic system. Substances that cause the state of dependence activate the dopaminergic neurons of the ventral tegmental area and cause emotionally positive hedonic reactions (Costardi et al., 2015; Keiflin \& Janak, 2015). At different stages of transformation of the state of dependence, various neurotransmitters and regulatory-modulatory factors are involved in the correction and maintenance of structural, functional and pathological changes. Much attention is paid to the dopamine-, glutamate-, GABA-, opioid-, cannabinoid- and nitrosergic systems (Harlan et al., 2018; Titkova et al., 2018). Over the past decade, research on neurosteroid hormones, their activity in the regulation of development, differentiation of the brain, reparative and cognitive functions, neurodegenerative processes in the nervous system have intensively developed. The special interest in the problem is caused by the fact that the research proved the existence of all the necessary conditions for the synthesis of steroid hormones directly in the brain tissues (Mellon et al., 2001). The study found that the regulatory functions of neuroactive steroids have structural and functional differences, and are realized both at the genomic and non-genomic levels through the interaction with cell membrane receptors (Ritsner \& Weizman, 2008; Osterlund et al., 2016). The development of pathological states of dependence on psychoactive substances was observed to be closely related to the system of neurosteroid regulation (Koob, 2013; Mons \& Beracochea, 2016). Koob et al. (2014) hypothesized that the development of pathological dependence is based on the dysregulation of key neurochemical elements of the cerebral stress system, which is a part of the system of emotional motivational reinforcement. The authors focused on the hypothalamic-pituitary-adrenal axis, which is modulated by the corticotropin-releasing hormone, and on the dynorphin-opioid mechanisms of the amygdala as the main factors which support the dysphoric emotional state associated with the state of dependence. Other studies have shown that glucocorticoids may also have a positive reinforcement effect (Rose et al., 2010); and neuroactive sex hormones and progesterone are involved in the formation of allostasis specific to alcohol dependence (Lenz et al., 2012; Berchenco et al., 2017; Peltier \& Sofuoglu, 2018). It was demonstrated that in the attempt to suppress the craving for alcohol, aerobic exercises (running in a wheel) can reduce alcohol consumption by laboratory animals (Ehringer et al., 2009; Brager \& Hammer, 2012). In our previous study we found decrease in craving for alcohol due to running in a wheel in most rats with alcohol dependence, which was accompanied by changes in serum and hippocampus BDNF concentrations depending on the duration of exercise. The 
research is focused on the nature of changes in the electrical activity of the emotional limbic-neocortical system of the brain, the content of neurosteroid hormones in the brain and serum of rats with alcohol dependence in the state after alcohol withdrawal and their transformation during the period of reduced craving for alcohol as the result of dosed physical load.

\section{Materials and methods}

The procedures with experimental animals were approved by the Commission on Ethics and Deontology of the SI "Institute of Neurology, Psychiatry and Narcology of the National Academy of Medical Sciences of Ukraine" and performed in accordance with the "General Ethical Principles of Animal Experiments" (Kyiv, 2011), "The procedure for conducting experiments on animals by scientific institutions" (No. 249 of 01.03.2012), and the Law of Ukraine "On protection of animals from cruel treatment" (No. 3447-IV of 21.02.2006).

The studies were conducted in 48 outbred white adult male rats weighing $210-280 \mathrm{~g}$. Alcohol dependence modeling was performed by consuming food containing $1 \mathrm{~mL}$ of $24.0 \%$ ethanol solution for two months. A single dose of ethanol was $1.25 \mathrm{mg} / \mathrm{kg}$ of body weight of rats. The state of alcohol dependence in animals was achieved as the result of long-term alcoholization, and its formation was controlled by a neuroethological test for the preference of alcoholic food (rats made choices between pieces of bread with or without ethanol). The vast majority of rats (96.2\%) chose pieces with ethanol. Withdrawal of alcohol was carried out over 7-10 days. Dosed physical load was achieved by running in a wheel for small animals (Galperin \& Tatarskij, 1967) for 30 minutes daily for $7-$ 10 days. Dosed physical load was applied both to rats after alcohol withdrawal and to the control group of comparison-intact animals (Fig. 1).

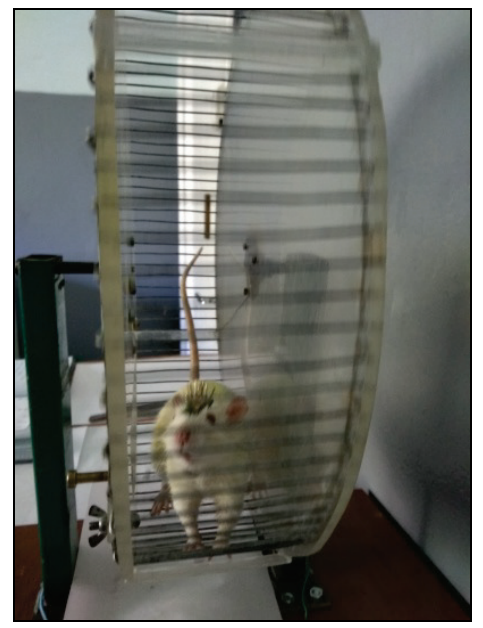

Fig. 1. Rat nunning in a wheel

Stereotactic operations of implantation of electrodes into the structures of the rat brain (dorsal hippocampus and amygdala) were performed under general anesthesia with sodium thiopental at the dose of $50 \mathrm{mg} / \mathrm{kg}$ of body weight. Implantation of long-term nichrome electrodes in fibreglass insulation (diameter of $100 \mathrm{~mm}$ ) was performed in accordance with the brain maps by Fifkova, Marshall (Buresh et al., 1962). Cortical nichrome electrodes were inserted into the epidural space of the frontal-parietal area, the reference electrode was placed into the bone of the nasal sinus. Bipolar recording of electrical activity of the brain and analysis of the absolute spectral power density of the electroencephalogram rhythms were performed on the computer-diagnostic complex "Neuron Spectrum +" (Ukraine, Spectromed LLC, 2009). The absolute spectral power density of the bioelectric signal was estimated in the following frequency ranges: delta $(0.5-4.0 \mathrm{~Hz})$, theta $(4.0-7.0 \mathrm{~Hz})$, alpha $(8.0-12.0 \mathrm{~Hz})$, beta $1(14.0$ $20.0 \mathrm{~Hz})$, beta $2(20.0-35.0 \mathrm{~Hz})$. Epochs without artifacts were used for the analysis (the duration of one epoch was $5 \mathrm{~s}$ ).

The content of progesterone, cortisol, testosterone and estradiol in the brain structures (hypothalamus, amygdala, hippocampus) as well as cortisol and testosterone in serum were determined in the rats with alcohol dependence and in rats with dosed physical load immediately after running in the wheel. Although corticosterone is the predominant glucocorticoid hormone in rats, cortisol is also synthesized and released in the blood and brain structures, and the sensitivity of mineralocorticoid and glucocorticoid receptors has similar parameters for both neurosteroids (Carroll \& Heath, 1975; Chapman et al., 2013; Gong et al., 2015; Samidurai et al., 2018). The review (Taves et al., 2011) provides an analysis of the methods for determining steroid hormones in brain tissues. We used the ethyl acetate extraction. For this purpose the brain structures were homogenized in $0.5 \mathrm{~mL}$ of $0.1 \mathrm{~N} \mathrm{HCl}, 1.5 \mathrm{~mL}$ of ethyl acetate was added, then the structures were shaken on a shaker for $15 \mathrm{~min}$ and frozen overnight at $-20^{\circ} \mathrm{C}$.

The unfrozen organic phase was decanted and evaporated in a water thermostat at $+56{ }^{\circ} \mathrm{C}$. The precipitate was dissolved in $0.2 \mathrm{~mL}$ of doubledistilled water and centrifuged at $7000 \mathrm{rpm}$ for $5 \mathrm{~min}$. The concentration of steroid hormones in the supernatant and blood serum was determined using the enzyme-linked immunosorbent assay kits: "Progesterone", "Cortisol", "Testosterone" and "Estradiol" (Granum, Ukraine). The optical density of the samples was measured on an analyzer Stat-Fax 2100 (Awareness Technology Inc., USA, 2010). The concentration of steroids in brain tissues was expressed in $\mathrm{pmol} / \mathrm{g}$ of wet weight, in serum - in nmol/L. The similar studies were carried out in control groups (non-alcoholic rats received the same dosed physical load and intact animals).

Statistical analysis of the obtained data was performed using the program "Statistica 6.0" (Statsoft Inc., USA, 2001) with the mean and standard deviation $(\mathrm{x} \pm \mathrm{SD})$ for each group. One-way analysis of variance ANOVA was used to detect statistically significant differences between groups. Differences were considered significant at $\mathrm{P}<0.05$ according to the Tukey test.

\section{Results}

Neuroethological testing of alcohol craving revealed that the longterm alcohol intake resulted in rats preferring alcoholic food to nonalcoholic food. There was a $100.0 \%$ dependence on alcohol in rats, which manifested itself in an instant reaction to alcoholic food, which the rats ate quickly (Fig. 2).

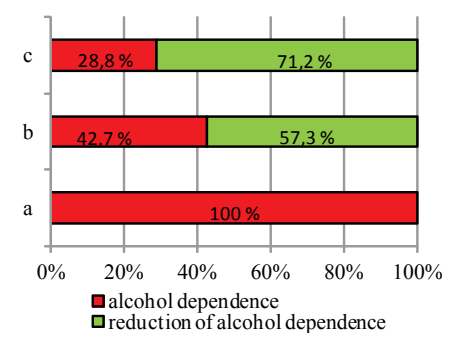

Fig. 2. The effect of dosed physical load on the craving to alcohol in rats in the test of the preference of alcoholic food to non-alcoholic: $a-$ alcohol dependence, $b-$ after 5 days of dosed physical load, $c$-after 7 days of dosed physical load

Consumption of the next dose of alcoholized food by rats with alcohol dependence led to the increase of inhibitory processes on the electroencephalograms of the neocortex, hippocampus and amygdala. The hippocampus played the pacemaker role in initiation of paroxysmal activity with generalization of paroxysmal process in the neocortex (Fig. 3).

Dosed physical load (5 days for 30 minutes with an intensity of 15 20 revolutions per minute) in the state of alcohol withdrawal led to decrease of alcohol motivation in $57.3 \%$ of the rats. The animals demonstrated a prolonged orientation - research reaction to situational afferentation with inhibition of purposeful movement towards alcoholic food and its partial consumption after presentation. On day 7 , dosed exercise led to suppression of alcohol motivation in $71.2 \%$ of the rats. The animals were in a comfortable condition and showed positive grooming reactions and lost interest in alcoholic food (Fig. 2). Suppression of alcohol motivation persisted the next day without dosed exercise use.

Changes in the electrical activity of the brain structures on the 5th day after dosed exercise were expressed in the suppression of delta-range paroxysmal complexes in the hippocampus and the enhancement of thetabiopotentials in the amygdala. The experiment has revealed a significant $(\mathrm{P}<0.05)$ increase in the spectral power of the theta range in the spectrogram of the amygdala (Fig. 4). Probably, such changes on the electroencephalogram of the amygdala occurred due to the manifestation of motiva- 
tional arousal as a consequence of alcohol withdrawal. On the 7th day after dosed physical load an increase in biopotentials of the fast frequencyes of the alpha- and beta-ranges in the electrical activity of the neocortex was detected as a reflection of the increased tonus of the neocortex (Fig. 3). A decrease $(\mathrm{P}<0.05)$ in the spectral power of the theta-range in the amygdala was also observed (Fig. 4).
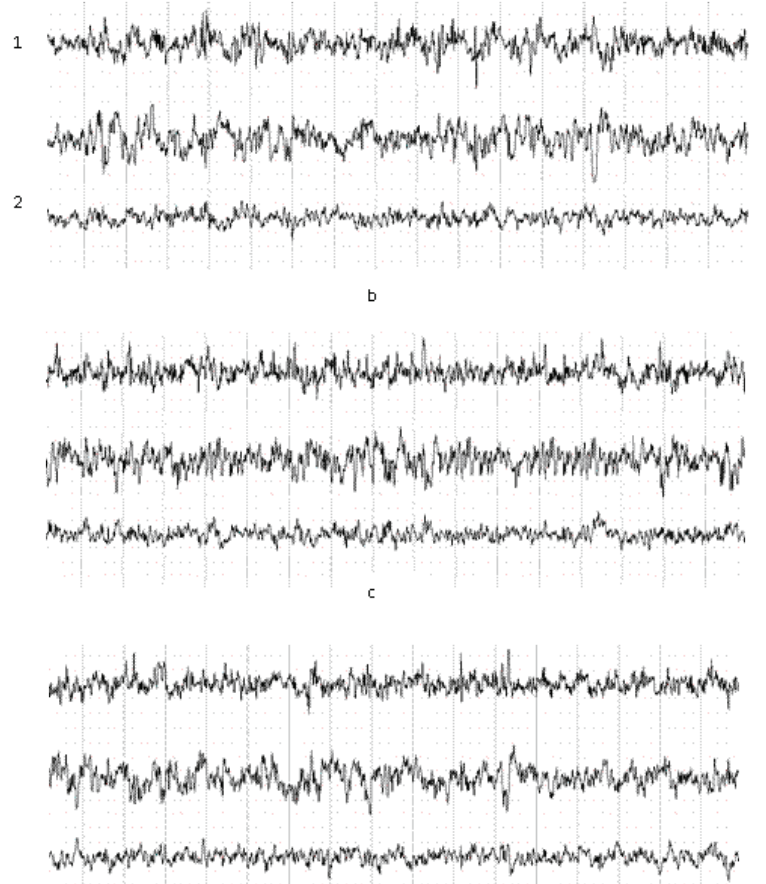

Fig. 3. Dynamics of electrical activity of brain structures in the rat No. 7 with alcohol dependence $(a)$, on the fifth $(b)$ and seventh $(c)$ day after dosed physical load 1 -neocortex, 2 - hippocampus, 3 - amygdala; calibrate signal $10 \mu \mathrm{V} / \mathrm{s}$

Formation of a short-term allostatic state during the period of abstinence usually ends up with the craving for the next dose of alcohol. In our study after 7-10 days of alcohol withdrawal the rats tested for the level of dependence revealed the preservation of their craving for alcohol. A study of the spectrum of neurosteroid hormone concentrations during this period showed that a few days after alcohol withdrawal the state of the rats could be described as stress-like. It was proved by a significant increase in serum cortisol concentration by $50.4 \%$ and a decrease in testosterone concentration by $35.8 \%$ compared to the level of intact animals (Fig. 5, Table 1).

\section{Table 1}

The content of steroid hormones in the brain structures and serum of intact rats $(\mathrm{x} \pm \mathrm{SD})$

\begin{tabular}{lcccc}
\hline Hormone & $\begin{array}{c}\text { Hypothalamus, } \\
\text { pmol/g of wet } \\
\text { weight }\end{array}$ & $\begin{array}{c}\text { Amygdala, } \\
\text { pmol/g of wet } \\
\text { weight }\end{array}$ & $\begin{array}{c}\text { Hippocampus, } \\
\text { pmol/g of wet } \\
\text { weight }\end{array}$ & $\begin{array}{c}\text { Serum, } \\
\mathrm{nmol} / \mathrm{L}\end{array}$ \\
\hline Cortisol & $8.8 \pm 5.6$ & $68.0 \pm 10.1$ & $55.4 \pm 7.8$ & $7.9 \pm 1.4$ \\
Testosterone & $130.5 \pm 19.0$ & $97.5 \pm 9.9$ & $89.7 \pm 12.7$ & $4.8 \pm 1.5$ \\
Progesterone & $7.3 \pm 1.3$ & $10.1 \pm 2.3$ & $8.4 \pm 1.4$ & - \\
Estradiol & $37.0 \pm 1.3$ & $28.6 \pm 2.5$ & $50.2 \pm 8.1$ & - \\
\hline
\end{tabular}

The state of alcohol withdrawal was accompanied by multidirectional changes in neurosteroid concentrations in the brain structures. In the hypothalamus the progesterone level was increased by $58.7 \%$ against intact rats without significant changes in the content of other hormones. Similar changes took place in the hippocampus: an increase in the concentration of progesterone by $34.4 \%$ compared to the level of intact rats. In the amygdala the opposite changes were revealed: a significant decrease in the content of progesterone by $44.2 \%$ against its value in intact rats and testosterone - by $24.1 \%$ against the level in intact animals. The level of cortisol on the 7-10th days of alcohol withdrawal did not change significantly in the examined structures of the brain (Fig. 5, Table 1).

The use of dosed physical load for 7 days led to a decrease in craving for alcohol in $71.2 \%$ of the rats (Fig. 2). In the serum, a significant $(\mathrm{P}<$
0.05 ) increase in cortisol concentration was recorded in both rats in the state of alcohol withdrawal and in animals that did not take alcohol, but the effects of dosed exercises and alcohol withdrawal were not summarized. A significant $(\mathrm{P}<0.05)$ decrease in the level of cortisol by $32.3 \%$ compared to its values in intact animals was observed in the hypothalamus of rats of the control group with dosed exercises. The effect of dosed physical load on the background of alcohol withdrawal on serum testosterone levels was unexpected: the concentration of the hormone was higher by $125.8 \%$ compared to its level in intact rats immediately after running in a wheel, while no significant changes in this index were registered immediately after dosed physical load in animals which did not take alcohol.
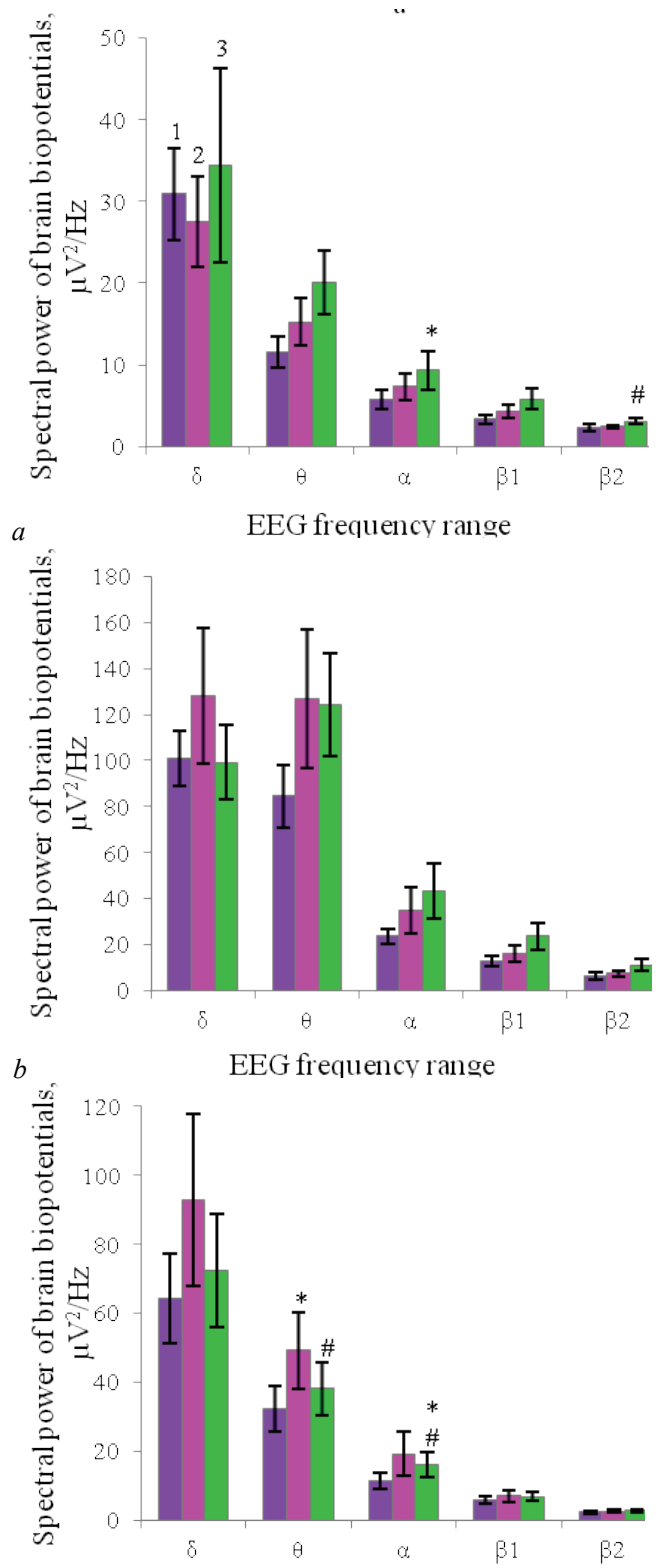

c EEG frequency range

Fig. 4. Influence of dosed physical load on the absolute spectral power density of the bioelectric signals of rhythms in the neocortex $(a)$, hippocampus $(b)$ and amygdala $(c)$ in rats with alcohol dependence: 1 -alcohol dependence; 2 - after 5 days of dosed physical load; 3 -after 7 days of dosed physical load; $\mathrm{x} \pm \mathrm{SD}, \mathrm{n}=10$; * $-\mathrm{P}<0.05$ compared to alcohol dependence, ${ }^{\#}-\mathrm{P}<0.05$ compared to the 5 th day of dosed physical load; here and in Figure 5 the significance of the differences between the samples was estimated with one-way analysis of variance ANOVA using the Tukey test

Intense functioning of the animal adaptation system was accompanied by a 3-fold increase in progesterone levels in the hypothalamus after dosed physical load on the background of alcohol withdrawal compared to this index in rats of the intact group, which was not observed in rats without alcohol consumption. At the same time recovery of the reduced 
by alcohol dependence progesterone and testosterone levels in the amygdala, and the decreased concentrations of progesterone by $28.1 \%$ and estradiol by $32.3 \%$ in the hippocampus against their content in intact rats were registered (Fig. 5, Table 1).

\section{Discussion}

Dosed physical load in rats with alcohol dependence causes a restructuring of the functional relationship between the structures of the emotional limbic-neocortical system of the brain depending on the duration of exercise. These changes are reflected in the level of alcohol motivation. Thirty-minute running in a wheel for 5 experimental days inhibits hypersynchronic paroxysmal activity on the EEG of cortical structures of the brain, which is an electrographic correlate of craving for alcohol (Huang et al., 2018; Veselovska et al., 2020). The increase in the spectral power of theta-rhythm in the amygdala, the brain structure, associated with negative emotional and motivational arousal, is a manifestation of functional tension as a consequence of the conflict of two motivations: craving for alcohol under alcohol withdrawal and interest in physical activity which suppresses negative emotional arousal (Seo, 2018). Further physical activity causes an increase in the tone of the neocortex as the result of the increased activation of the ascending pathways of the midbrain reticular formation, along with the suppression of alcohol drive. Thus, the primary target of dosed physical load is the phylogenetically ancient cortical structures of the brain (hippocampus and amygdala) with subsequent involvement of the neocortex.

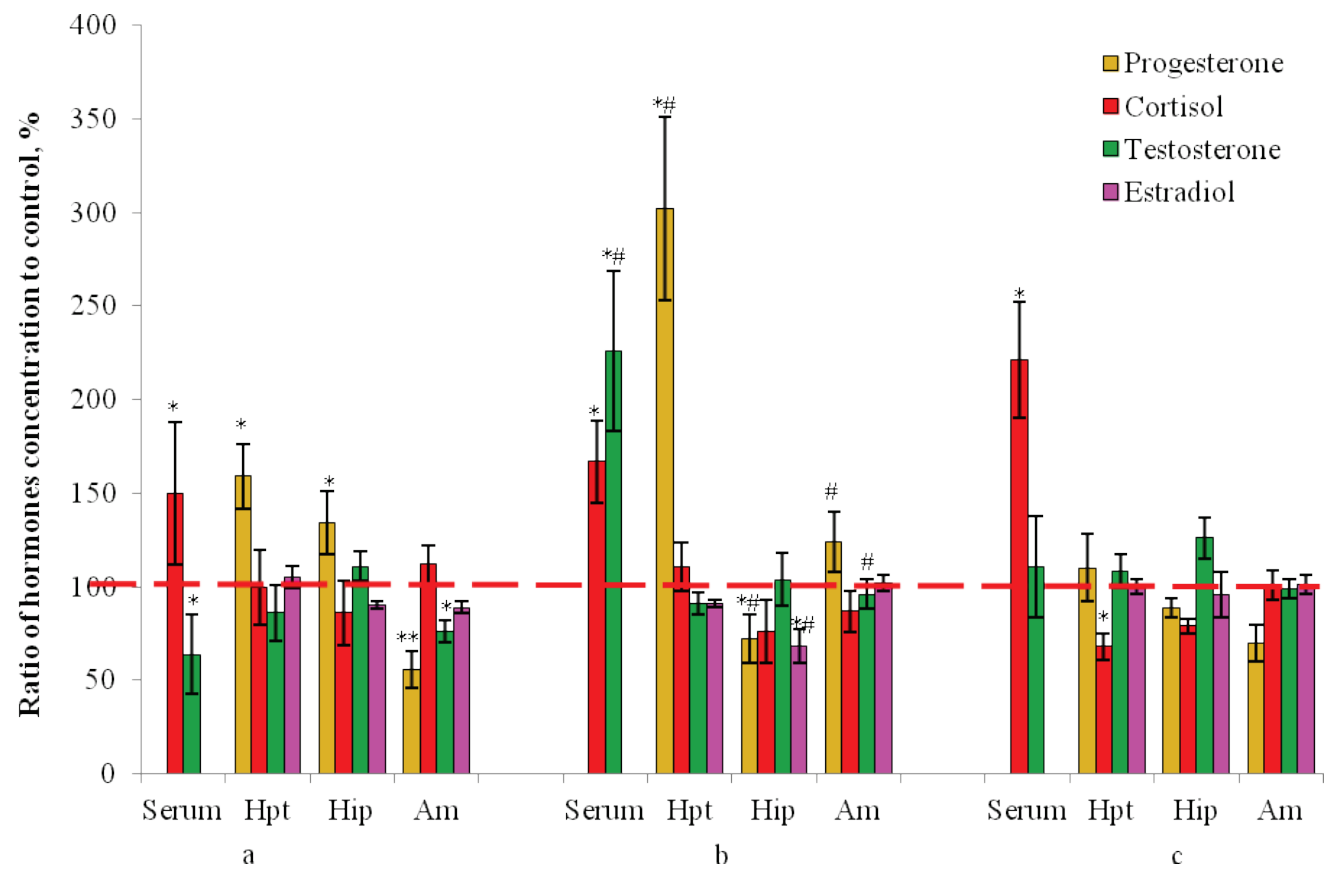

Fig. 5. Changes in the concentration of progesterone, cortisol, testosterone and estradiol in the serum (Serum), hypothalamus (Hpt), hippocampus (Hip) and amygdala (Am) of rats with alcohol dependence, in the state of alcohol withdrawal with dosed physical load and in animals after dosed physical load as a percentage to the level of intact rats (100.0\%): $a$-alcohol dependence, $b-$ dosed physical load on the background of alcohol withdrawal, $c$ - dosed physical load; $\mathrm{x} \pm \mathrm{SD}, \mathrm{n}=30 ; *-\mathrm{P}<0.05, * *-\mathrm{P}<0.01$ when compared with intact animals; ${ }^{*}-\mathrm{P}<0.05$ when compared with rats with alcohol dependence

Based on the known facts about the influence of neurosteroids on the development of the embryonic brain with the formation of neuronal networks associated with emotionally motivational behaviour, it was of interest to identify the implementation of these manifestations in the changes of the neuronal and peripheral hormone spectrum under alcohol dependence and its correction. Our study has demonstrated the varying degrees of preservation and regulatory sensitivity of peripheral mechanisms of cortisol synthesis in the adrenal glands and testosterone in the testes of male rats under the influence of dosed physical load on the background of the alcohol withdrawal after prolonged alcohol consumption. The fact of existence of functional tension in the adaptation system of the organism under physical loads was demonstrated as well. It is well known that alcohol dependence, as well as the alcohol withdrawal syndrome is accompanied by an increase in the concentration of glucocorticoids in the blood (Rose et al., 2010; Osterlund et al., 2016). Dosed exercise at the initial stage of run training in a wheel can have an imposed character, but later rats get used to it and run on their own. Therefore, the stress-like increase in cortisol levels in the serum of rats may be regarded as the result of increased motor activity which has an adaptive nature. The fact that the effects of alcohol withdrawal and dosed exercise have not been summarized may indicate that both factors have a strong impact on the adrenal mechanisms of regulation of glucocorticoid synthesis, more pronounced under physical loads. The content of cortisol in the hypothalamus of rats of both groups with alcohol dependence did not differ significantly from the level of intact animals. In contrast, in the control group with dosed physical loads (without alcohol intake) the level of glucocorticoid was signifi- cantly lower, which can help maintain the high level of the hormone in the blood by weakening the negative feedback from the cortisol in the hypothalamus. Because glucocorticoids are not accumulated in the tissues where they are synthesized (Walker, 2015), the findings indicate a constant activation of the hypothalamic-pituitary-adrenal system of adaptation.

Through non-genomic mechanisms glucocorticoids promptly regulate (diminish) the secretion of adrenocorticotropic hormone by pituitary corticotropic cells which allows synchronization of the rhythms of secretion of these hormones under normal operating conditions. Under prolonged enhanced loading on the system of adaptation, the central mechanisms of regulation become stronger at the level of the hypothalamus. Increased synthesis and release of the corticotropin-releasing hormone lead to transformation of the pulsatile nature of glucocorticoid secretion into a constantly increased release of the hormone (Gjerstad et al., 2018). According to the data (Koob, 2013) the corticotropin-releasing hormone is a leading factor in the mechanisms of formation of a stable pathological state under alcohol dependence. In one study (Osterlund et.al., 2016) it was directly demonstrated that rapid negative regulatory feedback caused by an increase in the blood glucocorticoids' concentration does not work at the background of the developed stress response. The data obtained by us confirm this fact.

Chronic tension in the hypothalamic-pituitary-adrenal system results in hormonal disregulation which was revealed only in the groups of rats with alcohol dependence and was not observed in the control group of animals with dosed exercise. On the 7th day of the alcohol withdrawal the hormonal disregulation was manifested in the increase of the progesterone 
content in the hypothalamus and multidirectional changes in its concentrations in the structures of the limbic system: an increase (in the hippocampus) and a decrease (in the amygdala). The described changes underwent transformations under the influence of dosed physical load. There was an even greater increase in progesterone levels in the hypothalamus and opposite changes in the structures of the limbic system: a decrease - in the hippocampus and an increase - in the amygdala (Fig. 5). It seems that progesterone is an important regulator of changes in the emotional and motivational status of rats dependent on alcohol.

Changes in the hormonal balance also affected the sex hormones of the brain. Testosterone contents were reduced in the amygdala and serum in rats with alcohol withdrawal. Dosed exercises stimulated hormone synthesis in the amygdala and on the periphery of the organism, but this effect was accompanied by a decrease in the estradiol content in the hippocampus (Fig. 5). The role of sex hormones in the regulation of motor activity was studied for a century, but only in recent decades have methodological opportunities made it possible to demonstrate the important and specific role of testosterone, estradiol and their receptors in maintaining body's activity. In the classical model of motor activity - running in a wheel - it was shown that the introduction of testosterone propionate or $17 \beta$-estradiol to mice restores motor activity of animals, which was reduced after gonadectomy, and the effect of testosterone was much more pronounced (Bowen et al., 2012). In our experiment we observed the opposite effect of the dosed physical load: the restoration of hormone levels reduced by chronic alcohol intake not only in the blood but also in the amygdala against the background of reduced estradiol content in the hippocampus. This suggests that testosterone levels, both central (in the amygdala) and peripheral, are not only important in maintaining the physical activity of rats, but are also the result of this activity. It may be a short-term release of the hormone after dosed exercise (we have not studied the long-term effects), but this indicates the presence of a significant compensatory resource for testosterone synthesis in animals with alcohol dependence in a state of alcohol withdrawal.

The effects of progesterone at a dose of $0.5 \mathrm{mg} / \mathrm{kg}$ body weight and the rat running in a wheel on the self-administration of a cocaine solution were studied (Zlebnik \& Saykao, 2014). The authors found a reduction in the animal's craving for the drug to be more effective when both factors were used. In our study it was shown that in the state of alcohol dependence on the background of alcohol withdrawal the level of progesterone in the brain changed unevenly: it was increased in the hypothalamus and hippocampus and decreased in the amygdala. Dosed exercise caused the opposite changes: a decrease in the concentration of the hormone in the hippocampus and normalization of its level in the amygdala (Fig. 5). Thus, progesterone has specific structurally dependent concentration changes both in the state of alcohol dependence and under the influence of dosed exercises, which gives reason to consider it as one of the key factors in allostatic restructuring of the functional state of animals under alcohol dependence.

Summarizing the data obtained, it can be argued that the state of alcohol withdrawal under the formed alcohol dependence is characterized by activation of the peripheral glucocorticoid link with a decrease in the concentration of testosterone in the serum. Hormonal imbalance in the structures of the central nervous system manifested in increased concentration of progesterone in hypothalamus and opposite changes in the structures of the limbic system: an increase - in the hippocampus with a decrease - in the amygdala it is accompanied by a decreased level of testosterone.

The amygdala is known to be the central structure of the limbic system that responds to negative emotional stimuli. There is evidence that progesterone and testosterone act as synergists at physiological concentrations and sex hormones can modulate the interaction of progesterone with membrane receptors: to enhance hormone receptor binding in the structures of the hypothalamus and to diminish these connections in the amygdala by testosterone (Caldwell et al., 2001; Cooke, 2006; Peters et al., 2015). These results indicate not only the special status of the amygdala in the emotional and motivational control of behaviour, but also the features of its neuroregulatory mechanisms (Ciocca et al., 2016).

The use of dosed physical load suppresses alcohol dependence with the formation of a new allostatic state, metabolic and regulatory support of which is largely determined by the specific neurochemical composition and functional connections of the amygdala. This is manifested in oppo- site changes in the neurosteroid regulatory system of the amygdala and hippocampus: a restoration of progesterone and testosterone concentrations in the amygdala and a decrease of progesterone level in the hippocampus. Further study of the mechanisms of the central neurosteroid regulation and their influence on allostasis may take their place in approaches to improving the efficiency of alcohol dependence correction.

\section{Conclusion}

The allostatic state of maintaining alcohol dependence is formed under the alcohol withdrawal situation. The nature of hormonal dysfunction is manifested in an increase of progesterone level in the hypothalamus and hippocampus and in a decrease of progesterone and testosterone contents in the amygdala against the background of functional tension of the peripheral glucocorticoid regulatory link and a decrease of testosterone concentration in serum. Dosed physical load leads to the suppression of alcohol motivation of rats with alcohol dependence and causes positive changes in the electrogenesis of the brain: inhibits the hypersynchronic paroxysmal activity of the amygdala and hippocampus and increases the tone of the neocortex. A new allostatic state of alcohol dependence attenuation is formed on the background of the accumulation of progesterone in the hypothalamus and restoration of its level and testosterone content in the amygdala, as well as a decrease of progesterone and estradiol in the hippocampus.

\section{References}

Berchenco, O. G., Titkova, A. M., Veselovs'ka, O. V., Shlakhova, A. V., Levicheva, N. O., \& Prichod'ko, O. O. (2017). Electrical activity of the cerebral structures and regulatory effects of $\mathrm{NO}$, steroid hormones, and $\mathrm{BDNF}$ in rats with experimental alcohol addiction. Neurophysiology, 4(3), 240-242.

Bowen, R. S., Knab, A. M., Hamilton, A. T., McCall, J. R., Moore-Harrison, T. L., \& Lightfoot, J. T. (2012). Effects of supraphysiological doses of sex steroids on wheel running activity in mice. Journal Steroids Hormonal Science, 3(2), 110-121.

Brager, A. J., \& Hammer, S. B. (2012). Impact of wheel running on chronic ethanol intake in aged Syrian hamsters. Physiology and Behavior, 107(3), 418-423.

Buresh, Y., Petran, M, \& Zakhar, I. (1962). Elektro-fiziologicheskiye metody issledovaniya [Electro-physiological research methods]. Izdatelstvo Inostrannoj Literatury, Moscow (in Russian).

Caldwell, J. D., Gao, G., Frasch, M., Jirikowski, G. F., \& Witt, D. M. (2001). Testosterone alters membrane binding of progesterone in male rat brains. European Journal of Anatomy, 5(1), 37-45.

Carroll, B. J., \& Heath, D. B. (1975). Corticosteroids in brain tissue. Endocrinology, 97, 290-300.

Chapman, K., Holmes, M., \& Seckl, J. (2013). 11ß-Hydroxysteroid dehydrogenases: Intracellular gate-keepers of tissue glucocorticoid action. Physiological Reviews, 93(3), 1139-1206

Ciocca, G., Limoncin, E., Carosa, E., Sante S. D., Gravina, G. L., Mollaioli, D., Gianfrilli, D., Lenzi, A., \& Jannini, E. A. (2016). Is testosterone a food for the brain? Sexual Medicine Reviews, 4(1), 15-25.

Cooke, B. M. (2006). Steroid-dependent plasticity in the medial amygdale. Neuroscience, 138(3), 997-1005.

Costardi, J. V., Nampo, R. A., Silva, G. L., Ribeiro, M. A., Stella, H. J., Stella, M. B., \& Malheiros, S. V. (2015). A review on alcohol: from the central action mechanism to chemical dependency. Revista da Associação Médica Brasileira, 61(4), 381-387.

Ehringer, M. A., Hoft, N. R., \& Zunhammer, M. (2009). Reduced alcohol consumption in mice with access to a running wheel. Alcohol, 43(6), 443-452.

Galperin, S. I., \& Tatarskij, N. E. (1967). Metodiki issledovaniya vysshej nervnoj deyatel'nosti cheloveka i zhivotnykh [Research methods of higher nervous activity of humans and animals]. Vysshaya Shkola, Moscow (in Russian).

Gjerstad, J. K., Lightman, S. L., \& Spiga, F. (2018). Role of glucocorticoid negative feedback in the regulation of HPA axis pulsatility. Stress, 21(5), 403-416.

Gong, S., Miao, Y.-L., Jiao, G.-Z., Sun, M.-J., Li, H., Lin, J., Luo, M. J., \& Taning, H. J. (2015). Dynamics and correlation of serum cortisol and corticosterone under different physiological or stressful conditions in mice. PLoS One, 10(2), e0117503.

Harlan, B. A., Becker, H. C., Woodward, J. J., \& Riegel, A. C. (2018). Opposing actions of CRF-R1 and CB1 receptors on VTA GABAergic plasticity following chronic exposure to ethanol. Neuropsychopharmacology, 43, 2064-2074.

Huang, Y., Mohan, A., De Ridder, D., Sunaert, S., \& Vanneste, S. (2018). The neural correlates of the unified percept of alcohol-related craving: A fMRI and EEG study. Scientific Reports, 8, 923-934. 
Keiflin, R., \& Janak, H. (2015). Dopamine prediction errors in reward learning and addiction: From theory to neural circuitry. Neuron, 88(2), 247-263.

Koob, G. F. (2013). Theoretical frameworks and mechanistic aspects of alcoho addiction: Alcohol addiction as a reward deficit disorder. Current Topics in Behavioral Neurosciences, 13, 3-30.

Koob, G. F., Buck, C. L., Cohen, A., Edwards, S., Park, P. E., Schlosburg, J. E., Schmeichel, B., Vendruscolo, L. F., Wade, C. L., Whitfield Jr., T. W., \& George, O. (2014). Addiction as a stress surfeit disorder. Neuropharmacology, 76, 370-382.

Lenz, B., Muller, C. P., Stoessel, C., Sperling, W., Biermann, T., Hillemacher, T., Bleich, S., \& Kornhuber, J. (2012). Sex hormone activity in alcohol addiction: Integrating organizational and activational effects. Progress in Neurobiology, 96, 136-163.

Mellon, S. H., Griffin, L. D., \& Compagnone, N. A. (2001). Biosynthesis and action of neurosteroids. Brain Research Reviews, 37, 3-12.

Mons, N., \& Beracochea, D. (2016). Behavioral neuroadaptation to alcohol: From glucocorticoids to histone acetylation. Frontiers in Psychiatry, 7, 165-173.

Osterlund, C. D., Rodriguez-Santiago, M., Woodruff, E. R., Newsom, R. J., Chadayammuri, A. P., \& Spencer, R. L. (2016). Glucocorticoid fast feedback inhibition of stress-induced ACTH secretion in the male rat: rate independence and stress-state resistance. Endocrinology, 157(7), 2785-2798.

Peltier, M. R., \& Sofuoglu, M. (2018). The role of exogenous progesterone in the treatment of males and females with substance use disorders: A narrative review. CNS Drugs, 32(5), 421-435.

Peters, S., Jolles, D. J., Van Duijvenvoorde, A. C., Crone, E. A., \& Peper, J. S. (2015). The link between testosterone and amygdala-orbitofrontal cortex connectivity in adolescent alcohol use. Psychoneuroendocrinology, 53, 117-126.
Ritsner, M. S., \& Weizman, A. (2008). Neuroactive steroids in brain function, behavior and neuropsychiatric disorders: Novel strategies for research and treatment. Springer.

Rose, A. K., Shaw, S. G., Prendergast, M. A., \& Little, H. J. (2010). The importance of glucocorticoids in alcohol dependence and neurotoxicity. Alcoholism: Clinical and Experimental Research, 34(12), 2011-2018.

Samidurai, M., Kang, H., Ramasamy, V. S., \& Jo, J. (2018). Impact of electrical stimulation on cortisol secretion in rat adrenal gland. Biochip Journal, 12, 216-221.

Seo, J. H. (2018). Treadmill exercise relieves stress-induced anxiety behavior in rats. Journal of Exercise Rehabilitation, 14(5), 724-730.

Taves, M. D., Ma, C., Heimovics, S. A., Saldanha, C. J., \& Soma, K. K. (2011). Measurement of steroid concentrations in brain tissue: Methodological considerations. Frontiers in Endocrinologi (Lausanne), 2, 1-13.

Titkova, A. M., Berchenko, O. G., Shlyahova, A. V., Veselovskaya, E. V., \& Prihodko, E. A. (2018). Vzaimodejstvija dofamina, oksida azota i testosterona v mozgovoj sisteme motivacionnogo podkreplenija krys s alkogolnoj zavisimostju pod vlijaniem donatora oksida azota [Interaction of dopamine, nitric oxide and testosterone in the brain system of motivational reinforcement in rats with alcohol dependence and under nitric oxide donator impact]. The Journal of V. N Karazin Kharkiv National University, Series Biology, 30, 92-98.

Veselovska, O. V., Shliakhova, A. V., Berchenko, O. G., \& Titkova, A. M. (2020) Neuroadaptation in the system of emotional reinforcement under alcohol dependence. Ukrainskyi Visnyk Psykhonevrolohii, 28(3), 22-25 (in Ukrainian).

Walker, J. J., Spiga, F., Gupta, R., Zhao, Z., Lightman, S. L., \& Terry, J. R. (2015). Rapid intra-adrenal feedback regulation of glucocorticoid synthesis. Journal of the Royal Society, Interface the Royal Society, 12, 20140875.

Zlebnik, N. E., Saykao, A. T., \& Carroll, M. E. (2014). Effects of combined exercise and progesterone treatments on cocaine seeking in male and female rats. Psychopharmacology, 231(18), 3787-3798. 